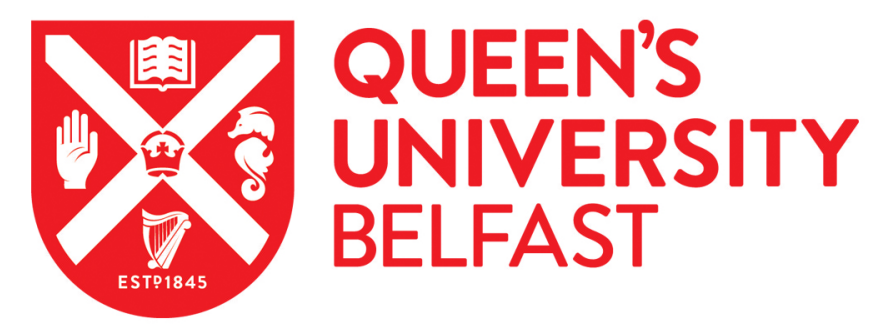

\title{
Mechanism of detonation stabilization in a supersonic model combustor
}

Cai, X., Deiterding, R., Liang, J., Mahmoudi, Y., \& Sun, M. (2021). Mechanism of detonation stabilization in a supersonic model combustor. Journal of Fluid Mechanics. https://doi.org/10.1017/jfm.2020.920[Opens in a new window]

Published in:

Journal of Fluid Mechanics

Document Version:

Peer reviewed version

Queen's University Belfast - Research Portal:

Link to publication record in Queen's University Belfast Research Portal

Publisher rights

(C) 2020 The Authors.

This work is made available online in accordance with the publisher's policies. Please refer to any applicable terms of use of the publisher.

\section{General rights}

Copyright for the publications made accessible via the Queen's University Belfast Research Portal is retained by the author(s) and / or other copyright owners and it is a condition of accessing these publications that users recognise and abide by the legal requirements associated with these rights.

Take down policy

The Research Portal is Queen's institutional repository that provides access to Queen's research output. Every effort has been made to ensure that content in the Research Portal does not infringe any person's rights, or applicable UK laws. If you discover content in the Research Portal that you believe breaches copyright or violates any law, please contact openaccess@qub.ac.uk. 


\section{Mechanism of detonation stabilization in a supersonic model combustor}

Xiaodong Cai ${ }^{1}$, Ralf Deiterding ${ }^{2}$, Jianhan Liang ${ }^{1 *}$, Yasser Mahmoudi ${ }^{3}$, Mingbo Sun ${ }^{1 *}$

${ }^{1}$ Science and Technology on Scramjet Laboratory

National University of Defense Technology, Changsha, 410073, China

${ }^{2}$ Aerodynamics and Flight Mechanics Research Group, University of Southampton

Boldrewood Campus, Southampton SO16 7QF, United Kingdom

${ }^{3}$ School of Mechanical and Aerospace Engineering, Queen's University Belfast

Belfast BT9 5AH, United Kingdom

*Corresponding author: jhleon@vip.sina.com,xd.cai.aero@outlook.com 


\section{Abstract:}

The present work studies numerically the quasi-steady propagation of a hydrogen/oxygen detonation in a supersonic model combustor consisting of a cavity and an expanding wall. The two-dimensional reactive compressible Navier-Stokes equations with one-step and two-species reaction model are solved using a hybrid sixth-order Weighted Essentially Non-Oscillatory-Centered Difference scheme combined with a structured adaptive mesh refinement technique. Results show that after the shutdown of the hot jet, the detonation wave is successfully stabilized quasi-steadily in the supersonic model combustor together with periodic fluctuations of the detonation front. The formation of the quasi-steady propagation of detonation in the model combustor is mainly due to the combined effects of (i) pressure oscillations generated in the cavity, which facilitate the detonation propagation, and (ii) lateral mass divergence brought by the expanding wall which can lead to detonation attenuation, and an unburned jet associated with large-scale vortices resulting from a Prandtl-Meyer expansion fan. This expansion fan is generated because of the expanding wall which can contribute to the detonation stabilization. It is found that for an incoming velocity lower than the Chapman-Jouguet value, a quasi-steady propagation of the detonation wave cannot be achieved. However, for incoming velocity higher than the Chapman-Jouguet value, a stabilization can be realized. This is effectively due to the formation of a periodic process including four stages of forward propagation, detonation attenuation, backward propagation and detonation bifurcation, indicating the influence of the supersonic model combustor on the overall process.

Key words: Detonation wave; Supersonic model combustor; Quasi-steady detonation propagation; Stabilization mechanism 


\section{Introduction}

Due to the superior performance at high Mach numbers ( $\mathrm{Ma} \geq 5.0)$, scramjets have become one of the first choices for hypersonic air-breathing propulsion systems. It is believed that the performance of the scramjet might be improved significantly if detonation-driven combustion is generated in supersonic flows because of the inherent theoretical advantage of detonation over deflagration combustion (Kailasanath 2008; Wolański 2013).

On the basis of the detonation-driven scramjet (DDS) (Cai et al. 2018a), numerical investigations have been carried out on detonation initiation and propagation in supersonic flows using a hot jet initiation (Cai et al. 2017; Cai et al. 2018b), which have indicated that maintaining the stabilization of a detonation wave in supersonic combustors is a great challenge that needs to be addressed. Lu and Braun (2014) have reported that for practical detonation-driven engines it is a key issue to be able to sustain a detonation wave in the combustor for a long duration when bringing the concept to reality.

It is critical to stabilize the detonation in supersonic combustors with limited length when it propagates at the Chapman-Jouguet $(\mathrm{CJ})$ state. In this regard, the first approach is to use cavities usually utilized as flameholders in supersonic combustors (Kang et al. 2011; Yeom et al. 2013; Tatman et al. 2013) to achieve detonation stabilization in supersonic flows. It is reported that the cavity can accelerate the propagation of the detonation wave in supersonic combustible mixtures which finally results in an overdriven detonation due to the enhancement of pressure oscillations caused by subsonic combustion in the cavity, and especially owing to the detonation wave interaction with the cavity a periodical process is generated to avoid detonation failure (Cai et al. 2018c). As a second approach, the expanding channels utilized in the actual propulsion combustors for 
maximizing thrust, has shown to be a promising method in sustaining the propagation of a detonation wave in supersonic flows. It is found that detonation sustainment can be maintained almost in the same position after the shutdown of the hot jet, suggesting that quasi-steady propagation of detonation can be achieved in this case for a given expansion angle; however, it should be noted that normally the quasi-steady state of detonation propagation can only be maintained for a limited time because the detonation may gradually attenuate and finally fail due to the Prandtl-Meyer expansion fan resulting from the expanding wall (Cai et al. 2019). Overall, for the two approaches, the cavity can lead to the formation of an overdriven detonation due to enhancement of pressure oscillations while the quasi-steady state of detonation propagation can be maintained for only a limited time because of the expanding wall.

In the present work, the reactive compressible Navier-Stokes (NS) equations are solved to identify the mechanism of detonation stabilization in a supersonic model combustor (Potturi \& Edwards 2015; Sun et al. 2008), which consists of an expanding wall and a cavity. Previous investigations (Cai et al. 2018c; Cai et al. 2019) have shown that both the cavity and expanding wall configurations can result in the generation of unburned jets, whose consumption and subsequent heat release are subjected to rapid turbulent mixing and diffusion. It is reported that the invisicd paradox can be solved with the inclusion of diffusive effect (Radulescu 2018) and the absence of small-scale turbulent interactions, normally not properly accounted for in detonation modelling using Euler simulations, leads to significantly lower burning rates than observed experimentally and hence does not permit detonation self-sustainment (Radulescu et al. 2005). Therefore, the utilization of the compressible NS equations is necessary to ensure the proper resolution of these small-scale turbulent interactions associated with diffusion and mixing effects for more accurate physical descriptions when the unburned jet is generated behind the detonation front. The present work also deploys a 
high-order hybrid Weighted Essentially Non-Oscillatory-Centered Difference (WENO-CD) scheme (Hill \& Pullin 2004; Pantano et al. 2007) utilizing the open-source program Adaptive Mesh Refinement Object-oriented C++ (AMROC) (Deiterding 2003; Deiterding 2009) based on an Structured Adaptive Mesh Refinement (SAMR) framework (Berger \& Oliger 1984). The overall approach of the hybrid WENO-CD method combines the robustness of WENO for discontinuity capturing with the benefit of a centered scheme with low numerical dissipation in smooth solution regions and the efficiency of SAMR, which has been validated meticulously in detonation simulations (Grogan \& Ihme 2015; Wang et al. 2018; Peng et al. 2018; Zhang et al. 2020). This work is part of an ongoing research program, aiming at fundamental understanding of stabilization of detonation in supersonic flows.

The remainder of this paper is organized as follows: Section 2 introduces the computational model, including the governing equations, the numerical methods, and the computational setup. Results and discussion are shown in Section 3, where the self-sustaining propagation of detonation in the supersonic model combustor, the corresponding stabilization mechanism, and the subsequent effects of the incoming velocity are further analyzed. Finally, Section 4 concludes the paper.

\section{Computational model}

\subsection{Governing equations}

The two-dimensional compressible NS equations with one-step chemistry model are utilized as governing equations, which are expressed as follows:

$$
\frac{\partial U}{\partial t}+\frac{\partial\left(F_{c o n v}-F_{d i f f}\right)}{\partial x}+\frac{\partial\left(H_{c o n v}-H_{d i f f}\right)}{\partial y}=S_{c h e m} .
$$


The vector of state is

$$
U=\left(\rho, \rho u, \rho v, \rho e, \rho Y_{1}\right)
$$

where $\rho, u, v, e$ and $Y_{1}$ are the total density, the velocity in the $x$-direction, the velocity in the $y$-direction, the total energy per unit mass and the mass fraction of the reactant, respectively.

The convective and diffusive fluxes are

$$
\begin{gathered}
F_{c o n v}=\left(\rho u, \rho u^{2}+p, \rho u v, \rho u e+u p, \rho u Y_{1}\right) \\
H_{c o n v}=\left(\rho v, \rho u v, \rho v^{2}+p, \rho v e+v p, \rho v Y_{1}\right), \\
F_{d i f f}=\left(0, \tau_{x x}, \tau_{x y}, u \tau_{x x}+v \tau_{x y}+k \frac{\partial T}{\partial x}+\rho h_{1} D_{1} \frac{\partial Y_{1}}{\partial x}+\rho h_{2} D_{2} \frac{\partial Y_{2}}{\partial x}, \rho D_{1} \frac{\partial Y_{1}}{\partial x}\right) \\
H_{d i f f}=\left(0, \tau_{y x}, \tau_{y y}, u \tau_{y x}+v \tau_{y y}+k \frac{\partial T}{\partial y}+\rho h_{1} D_{1} \frac{\partial Y_{1}}{\partial y}+\rho h_{2} D_{2} \frac{\partial Y_{2}}{\partial x}, \rho D_{1} \frac{\partial Y_{1}}{\partial y}\right),
\end{gathered}
$$

and the reactive source term is

$$
S_{\text {chem }}=\left(0,0,0,0,0, \dot{w}_{1}\right) .
$$

Here, $\tau$ is the stress tensor; $h_{1}$ and $h_{2}$ are the enthalpies of the reactant and product, respectively; $\dot{w}_{1}$ is the mass production rate of the reactant. $\mu, k, D_{1}, D_{2}$ are the mixture viscosity, the thermal conductivity, and the mass diffusivities of the reactant and product, respectively. $e$ is defined as

$$
e=\frac{p}{\rho(\gamma-1)}+\frac{\left(u^{2}+v^{2}\right)}{2}+Y_{1} q
$$

where $q$ is the heat release per unit mass. The stresses read

$$
\tau_{x x}=\mu\left(\frac{4}{3} \frac{\partial u}{\partial x}-\frac{2}{3} \frac{\partial v}{\partial y}\right), \tau_{x y}=\tau_{y x}=\mu\left(\frac{\partial v}{\partial x}+\frac{\partial u}{\partial y}\right) \text { and } \tau_{y y}=\mu\left(\frac{4}{3} \frac{\partial v}{\partial y}-\frac{2}{3} \frac{\partial u}{\partial x}\right) .
$$

For the two species, the calorically perfect model

$$
\gamma=\gamma_{1}=\gamma_{2}, p=\rho R T, R=R_{1}=R_{2}
$$


is used. The mass fraction production rates are given as follows:

$$
\dot{w}_{1}=-\dot{w}_{2}=-\rho Y_{1} A \exp \left(-\frac{E_{a}}{R T}\right) .
$$

Currently, it is computationally very expensive to conduct high-resolution multi-dimensional detonation simulations solving the compressible NS equations with detailed chemistry. The Arrhenius law that relates chemical reaction rates to temperature variation is widely used as the simplest mode for detonation simulations (Romick, Aslam \& Powers 2012; Teng, Jiang \& Ng 2014; Shen \& Parsani 2017; Xiao \& Oran 2019). Here the reaction model (Bane, Ziegler \& Shepherd 2010) is selected and fitted to the physical parameters of a $\mathrm{H}_{2} / \mathrm{O}_{2}$ detonation initially at $T=300 \mathrm{~K}$ and $P=6.67 \mathrm{kPa}$ with the corresponding $\mathrm{CJ}$ velocity of $V_{C J}=1587.84 \mathrm{~m} / \mathrm{s}$. In order to make an approximate chemistry and transport model with the single Arrhenius rate equation, constant specific heat and temperature-dependence transport, the Zel'dovich-von Neumann-Döring (ZND) detonation solution is obtained with a detailed chemistry using a high-temperature extension of the GRI30 mechanism in Cantera and the SDToolbox (Kao \& Shepherd 2008). The Arrhenius rate activation energy, pre-exponential factor, heat release and specific heat ratio are chosen by matching the CJ speed and the von Neumann pressure at the beginning of the ZND detonation. This defines a marginally stable detonation with the corresponding average detonation cell of $\lambda=25 \mathrm{~mm}$ (Ziegler et al. 2011). The thermodynamic properties are shown in Table 1.

Table 1 Thermodynamic parameters.

\begin{tabular}{ccc}
\hline Parameters & Values & Unit \\
\hline$T_{\infty}$ & 300 & $\mathrm{~K}$ \\
$p_{\infty}$ & 6.67 & $\mathrm{kPa}$ \\
$\rho_{\infty}$ & 0.077552 & $\mathrm{~kg} / \mathrm{m}^{3}$ \\
$\gamma$ & 1.29499 & - \\
\hline
\end{tabular}


W

Heat release $(q)$

Activation energy $\left(E_{a}\right)$

Pre-exponent factor $(A)$
0.029

54000

30000

$6 \times 10^{5}$ $\mathrm{kg} / \mathrm{mol}$

$\mathrm{J} / \mathrm{mol}$

$\mathrm{J} / \mathrm{mol}$

$\mathrm{s}^{-1}$

The temperature and pressure at the end of the ZND reaction zone are approximately $2500 \mathrm{~K}$ and $1.01325 \times 10^{5} \mathrm{~Pa}$, respectively, which gives the transport parameters for the reaction model: $T_{r e f}=$ $2500 \mathrm{~K}, \mu_{r e f}=1.07 \times 10^{-4} \mathrm{~Pa} \cdot \mathrm{s}, k_{r e f}=0.148 \mathrm{~W} /(\mathrm{m} . \mathrm{K}), D_{1 r e f}=5.5 \times 10^{-4} \mathrm{~m}^{2} / \mathrm{s}, D_{2 r e f}=6.4 \times 10^{-4} \mathrm{~m}^{2} / \mathrm{s}$. The viscosity, conductivity and mass diffusivity are selected by matching the general trends and values at the end of the ZND reaction zone between the simplified model and when employing a detailed reaction model. Note that a local Reynolds number $\operatorname{Re}\left(=\frac{\rho v l_{1 / 2}}{\mu}\right)$ for the mixing along a shear layer behind the detonation front can be defined using the density $(\rho)$, local velocity $(v)$, viscosity $(\mu)$ and the half-reaction zone length $l_{1 / 2}$. The Sutherland model is utilized for the viscosity and conductivity while the mass diffusion values are derived from a simple expression that includes the inverse dependence on pressure, i.e.,

$$
\begin{gathered}
\frac{u}{u_{\text {ref }}}=\left(\frac{T}{T_{\text {ref }}}\right)^{\frac{5}{2}}, \frac{k}{k_{\text {ref }}}=\left(\frac{T}{T_{\text {ref }}}\right)^{\frac{5}{2}}, \\
\frac{D_{1}}{D_{\text {lref }}}=\left(\frac{T}{T_{\text {ref }}}\right)^{\frac{5}{2}} \frac{p_{a t m}}{p}, \frac{D_{2}}{D_{2 \text { ref }}}=\left(\frac{T}{T_{\text {ref }}}\right)^{\frac{5}{2}} \frac{p_{a t m}}{p} .
\end{gathered}
$$

\subsection{Computational setup}

The supersonic flow runs from right to left in the model combustor, as shown in Fig. 1. The length and height of the channel are $X_{1}=75 \mathrm{~mm}$ and $Y_{1}=25 \mathrm{~mm}$, respectively. The width of the hot jet used for the detonation initiation is $X_{2}=4 \mathrm{~mm}$ and the distance from the hot jet to the front edge of the cavity is $X_{3}=5 \mathrm{~mm}$. 


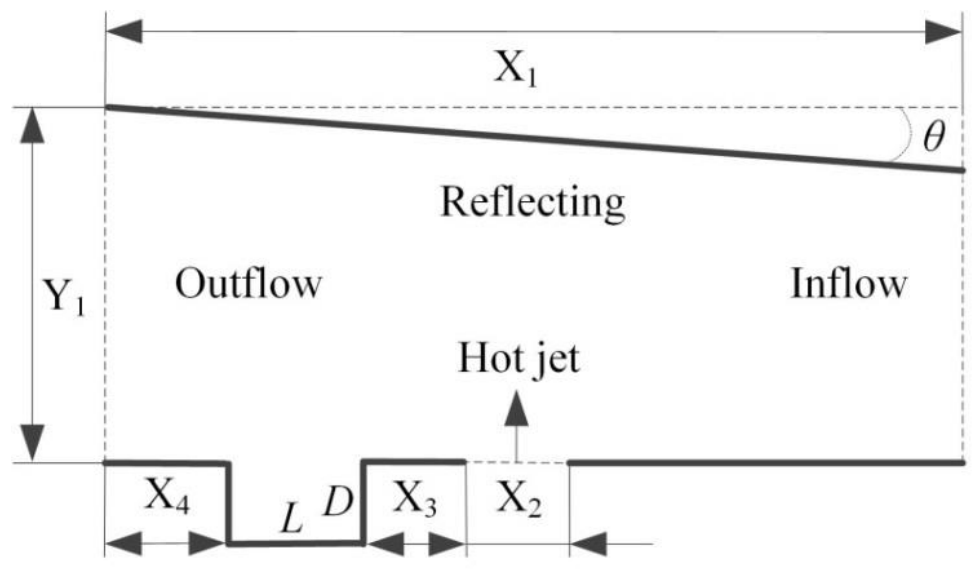

Fig. 1 Schematic sketch of the computational domain.

Downstream of the hot jet is a cavity with the width and depth of $L=20 \mathrm{~mm}$ and $D=10 \mathrm{~mm}$, respectively. The distance from the rear edge of the cavity to the outflow boundary is $X_{4}=15 \mathrm{~mm}$. An initial expansion angle of $\theta=4^{\circ}$ is employed for the expanding wall. It is found that for this given expansion angle detonation sustainment can be maintained in supersonic flows in the same position after the shutdown of the hot jet (Cai et al. 2019).

The level-set technique for chemically reactive flows (Deiterding 2009) is employed for the upper expanding channel section. The right boundary adopts the inflow condition and an ideal outflow condition implemented by constant value extrapolation of the interior data into the ghost cells is imposed on the left boundary. Reflecting boundaries with slip wall conditions are used on the upper and lower walls. In the viscid case, a turbulent boundary layer would develop along the inflow channel walls and its thickness will strongly depend on the length of the inflow section. Especially, at the start of the expansion section, the boundary layer will mostly detach, migrate partially into the channel interior and will only re-establish at the end of the expansion section. The process is rather complicate and can only accurately be described by simulating the entire combustion chamber. In order to eliminate this complexity and to be able to focus the present study just on detonation stabilization in the supersonic model combustor, we have opted to use the viscid model with slip 
boundary conditions in our investigations for not having to deal with boundary layers produced otherwise in the inflow section. At the lower wall, a small inflow condition is considered, representing a hot jet. The inflow parameters of the hot jet are set to the values of the CJ state of a $\mathrm{H}_{2} / \mathrm{O}_{2}$ detonation under the condition of pressure $6.67 \mathrm{kPa}$ and temperature $300 \mathrm{~K}$. The injection velocity is given as the sonic speed to make it a chocked flow. The detailed information of the hot jet is shown in Table 2. In order to control the injection duration of the hot jet, the parameter "time" is also considered when dealing with the boundary condition. After detonation initiation is realized successfully, the hot jet is switched off and the inflow condition is changed to a reflecting wall.

Table 2 Thermodynamic properties of the hot jet.

\begin{tabular}{ccc}
\hline Parameters & Values & Unit \\
\hline Pressure & 86376 & $\mathrm{~Pa}$ \\
Temperature & 1943.8 & $\mathrm{~K}$ \\
Density & 0.155 & $\mathrm{~kg} / \mathrm{m}^{3}$ \\
Velocity & 850 & $\mathrm{~m} / \mathrm{s}$ \\
Energy & 349280 & $\mathrm{~J} / \mathrm{mol}$ \\
Mass fraction of reactant $\left(Y_{1}\right)$ & 0.0088 & - \\
Mass fraction of product $\left(Y_{2}\right)$ & 0.9912 & - \\
\hline
\end{tabular}

\subsection{Numerical methods}

The hybrid sixth-order WENO-CD scheme ( Hill \& Pullin 2004; Pantano 2007), which has been verified previously (Cai et al. 2017; Ziegler et al. 2011), consists of two components: a finitedifference sixth-order WENO scheme to be used at discontinuities and a conservative sixth-order CD scheme for smooth-solution regions. Through a switch based on a shock-based detection technique (Lombardini 2008), the hybrid WENO-CD scheme can combine both the advantages of WENO and CD schemes: regions of strong discontinuities are approximated by the WENO scheme, while the 
$\mathrm{CD}$ scheme is used within regions of smooth flow, thus minimizing numerical dissipation to the extent possible.

Due to the stability properties of explicit integration schemes, the preferred practical method with the ability of inexpensive time adaptation in SAMR is Runge-Kutta methods of third or higher order. In the present work, the optimal third-order strong stability preserving (SSP) Runge-Kutta scheme (Gottlieb, Ketcheson \& Shu 2009) is used in combination with time-splitting and the fourthorder accurate semi-implicit A-stable generalized Runge-Kutta method of fourth order (GRK4A) method ( Kaps \& Rentrop 1979) for source term integration.

\subsection{Grid resolution analysis}

The initial grid resolution is $6.25 \times 10^{-5} \mathrm{~m}$. Three different cases of SAMR implementation are utilized for the resolution analysis. The detailed information for the three cases is shown in Table 3 .

Table 3 Mesh refinement parameters.

\begin{tabular}{ccc}
\hline Case 1 & Case 2 & Case 3 \\
3 levels & 4 levels & 5 levels \\
$(2,4)$ & $(2,2,4)$ & $(2,2,2,4)$ \\
$\Delta_{\min }=7.8 \times 10^{-6} \mathrm{~m}$ & $\Delta_{\min }=3.9 \times 10^{-6} \mathrm{~m}$ & $\Delta_{\min }=1.95 \times 10^{-6} \mathrm{~m}$ \\
\hline
\end{tabular}

Fig. 2 shows the typical structure of the double Mach stems (DMR) during the initiation through shock reflections induced by the hot jet using density contours, numerical schlieren and the corresponding mesh refinements. It is observed that the overall patterns of the Mach stems associated with the primary and secondary triple points, slip lines emitting from the triple points, are identical for all three cases in Fig. 2(a)(b)(c) and Fig. 2(d)(e)(f). Especially, these characteristic structures are all captured by the highest refinement levels as shown in Fig. 2(g)(h)(i), indicating that the refinement strategy established in the simulations can satisfy the requirements. 


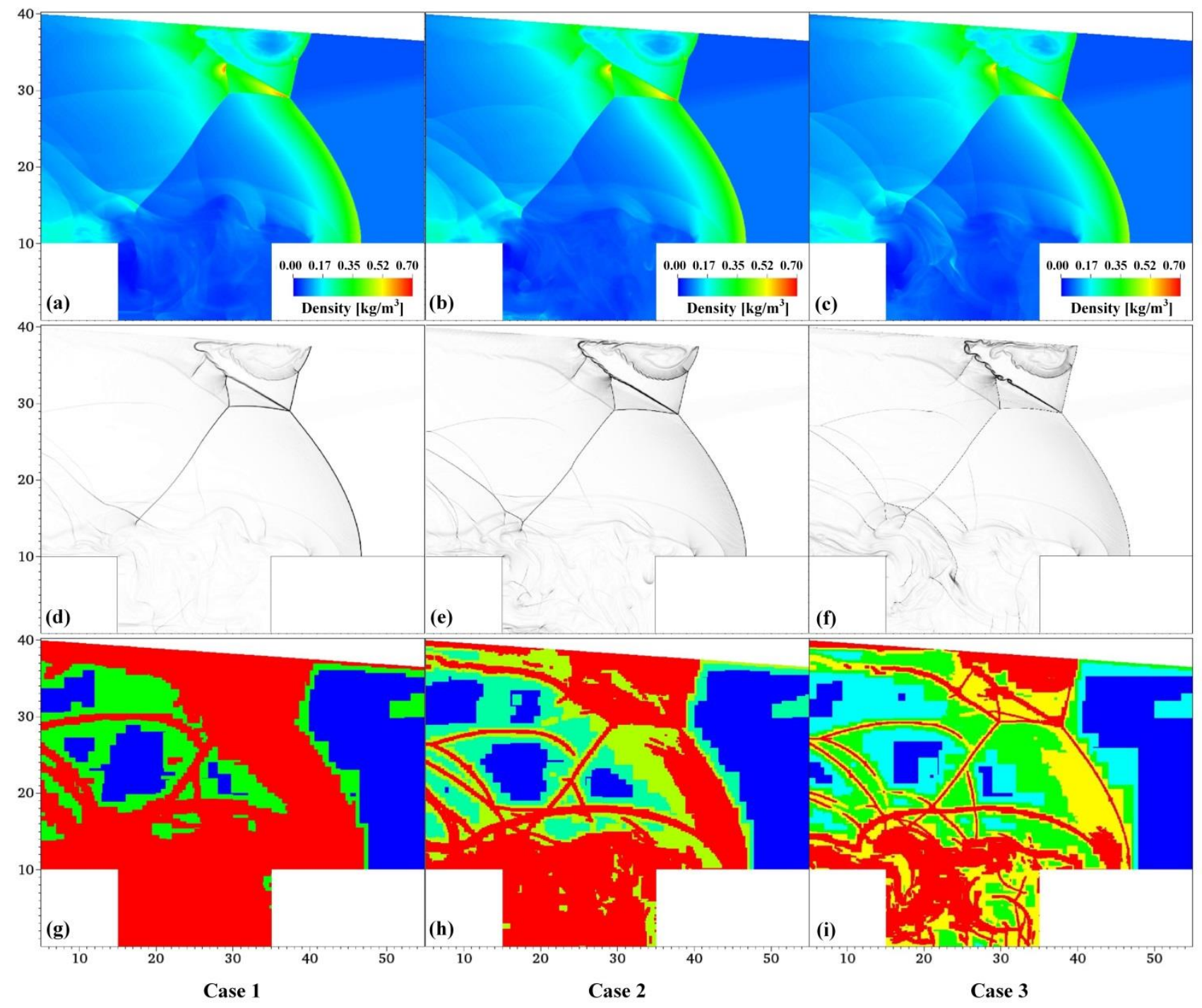

Fig. 2 Three refinement cases, (a) Case 1, (b) Case 2, (c) Case 3.

Fig. 3 shows the trajectory of the shock front and pressure oscillations behind the induced bow shock front versus time for the three cases. In Fig. 3(a), it is observed that the three curves are almost coupled together without obvious differences. In Fig. 3(b) the pressure curves show slightly staggered positions, especially for Case 1 at approximate $t=195 \mu \mathrm{s}$. Nevertheless, the curves for Case 2 and Case 3 are basically consistent.

As a compromise between the computational cost and resolved resolution, the four-level mesh refinement of Case 2 is adopted in the following simulations. 

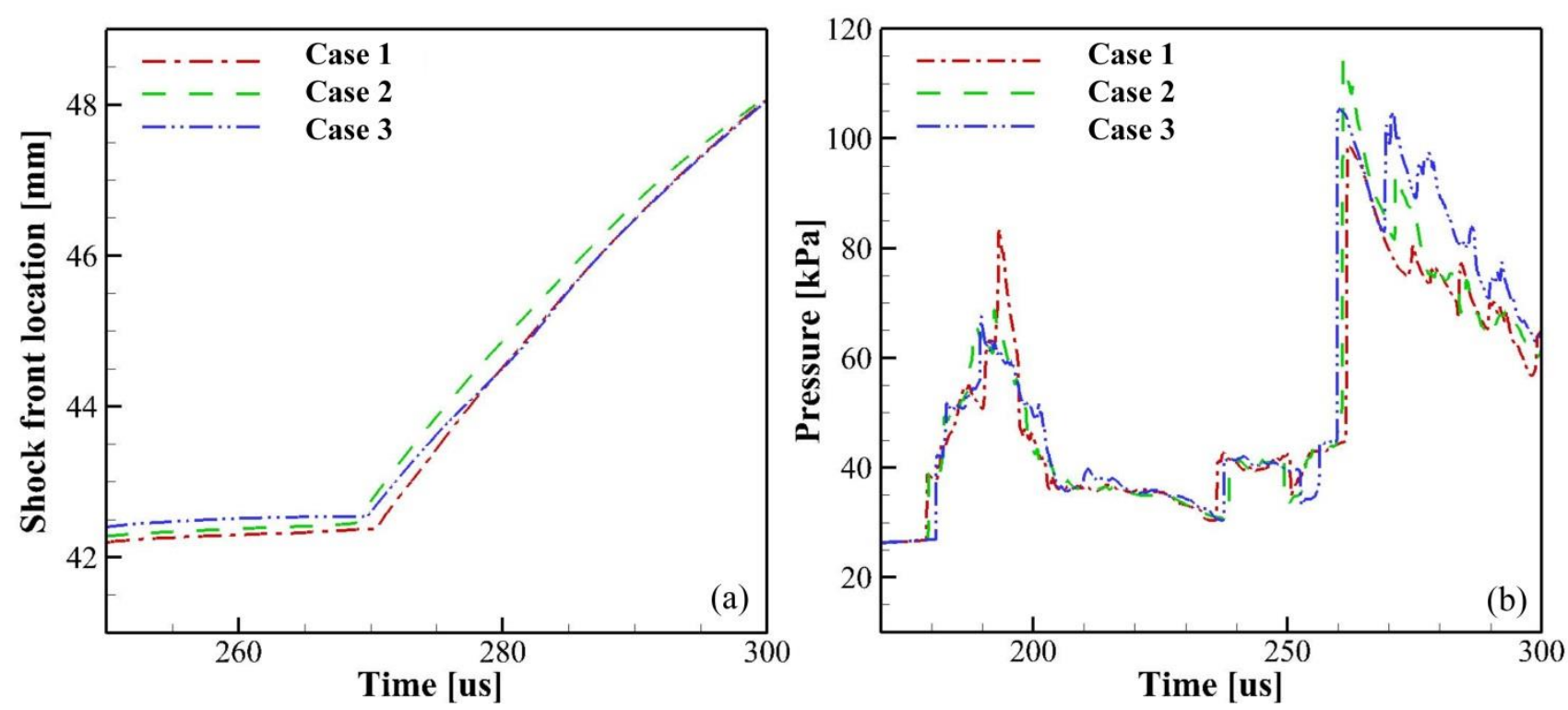

Fig. 3 The trajectory of the shock front and pressure oscillations versus time.

It is reported that the viscous scale is the smallest among the three diffusive scales (viscous shear layer, thermal heat conduction layer and mass diffusion layer) (Ziegler et al. 2011). The highest grid resolution in Case 2 of $\Delta_{\min }=3.9 \times 10^{-6} \mathrm{~m}$ corresponds to $525 \mathrm{Pts} / \mathrm{HRL}$ (points per half reaction zone length). Under this resolution in Case 2, about 20 cells can be placed within the viscous scale, hence indicating that these diffusive scales can be fully resolved in the present simulations. It should be noted that in two-dimensional high-resolution computations, the details of the turbulent structures such as vortex stretching are not necessarily resolved, and thus pseudo-DNS is being performed.

\section{Results and discussion}

\subsection{Self-sustaining propagation}

Fig. 4 shows the detonation propagation in the supersonic model combustor after the hot jet is shut down. It is observed in Fig. 4(a) that a small unburned jet is generated behind the detonation front due to the interaction of the Mach stem with the Prandtl-Meyer expansion fan. A detached shear layer is generated near the unburned jet owing to the triple point collision on the expanding wall. At 
this moment, only one triple point is observed on the detonation front.

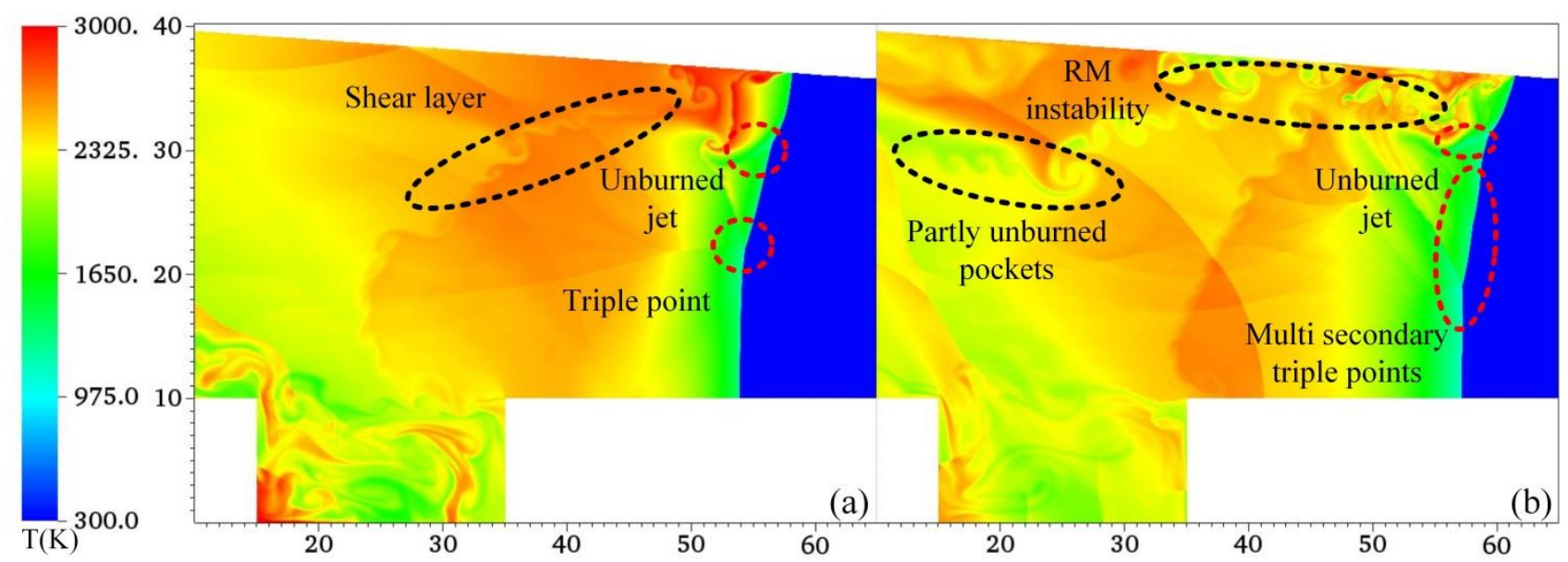

Fig. 4 Temperature contours showing detonation evolution after the shutdown of the hot jet, (a)

$$
t=345 \mu \mathrm{s}, \text { (b) } t=405 \mu \mathrm{s} \text {. }
$$

Although the hot jet is shut down, the detonation still continues its forward propagation for a while until it reaches the farthest position approximately at $X=58.6 \mathrm{~mm}$, where the hot jet effect is fully dissipated, as shown in Fig. 4(b). In the expanding wall region, the flow behind the detonation front is subsonic, thus the detonation experiences a lateral mass divergence (Radulescu \& Borzou 2018) brought by the area expansion. During this period, the detonation wave begins to attenuate gradually which leads to a weaker detonation as well as appearance of multiple secondary triple points as observed on the detonation front in Fig. 4(b). Maxwell et al. (2017) have reported that the triple point is a location of high temperature and pressure due to shock compression from multiple waves as well as a source of enhanced turbulent mixing. These triple points can give rise to slip lines further developing into shear layers that are susceptible to the Kelvin-Helmholtz (KH) instability (Massa, Austin \& Jackson 2007), hence acting to enhance the turbulent mixing between the unburned pockets and burned gases (Gamezo, Ogawa \& Oran 2007; Oran \& Gamezo 2007). It is seen in Fig. 4(b) that near the expanding wall, large-scale vortices are produced due to the interaction between the unburned jet and highly unstable shear layers emitted from secondary triple points. 
Some partly unburned pockets are even observed away from the detonation front after separating from the main unburned jet. These large-scale vortices can enhance the turbulent mixing between the unburned pockets and burned product, facilitating the consumption of the unburned pockets.

Globally, the Reynolds number is estimated using the average density $(\rho)$ and velocity $(v)$ at the top and bottom of the shear layer behind the triple point. Here, the Reynolds numbers in Figs. 4(a) and $4(\mathrm{~b})$ are $\operatorname{Re}=5 \times 10^{4}$ and $\operatorname{Re}=1 \times 10^{5}$, respectively. Dimotakis (2005) has reported that $\operatorname{Re}=10^{5}$ is an order of magnitude larger than the typical value for the onset of turbulence in mixing layers. The turbulence in detonations arises primarily from the jetting action of the slip lines and the $\mathrm{KH}$ instability along the shear layers (Radulescu et al. 2005). Therefore, after the shutdown of the hot jet, the flow field around the unburned jet associated with the highly unstable shear layers behind the detonation front, is in the turbulent regime after the detonation wave reaches the farthest position. This can further accelerate the consumption of the unburned jet by turbulent mixing.

As shown in Fig. 5, the successive four frames properly illustrate the oscillatory quasi-steady detonation propagation, where the detonation front oscillates almost at the same position of approximate $X=56.5 \mathrm{~mm}$. Near the expanding wall the structure of a shock-induced combustion wave is generated, as shown in Fig. 5(a). Behind the shock-induced combustion there are a series of large-scale vortices, and two main triple points are observed on the detonation front, propagating toward the lower wall. Therefore, the overall stabilization configuration is a combination of the detonation wave in the lower part and shock-induced combustion in the upper part. After further propagation, the triple points begin to move upward upon the collision with the lower wall. A small unburned jet is clearly observed at this moment as shown in Fig. 5(b), which is larger than that in Fig. 5(a). The large-scale vortex near the expanding wall is further dissipated due to the diffusion and mixing effects compared with that in Fig. 5(a) and further vanishes almost entirely in Fig. 5(c). 
Owing to the triple point collisions, detached shear layers are generated and subsequently interact with the large-scale vortices. With its extension of the newly generated unburned jet, vortices are produced along the unburned jet due to the KH instability as seen in Fig. 5(d). After the interaction with the highly unstable shear layers emitted from the secondary triple points, these small-scale vortices gradually become large, thus accelerating the heat release through consumption of the unburned jet due to the enhanced diffusion and mixing effects. Results shown in Fig. 5 illustrate a fully periodic process of quasi-steady detonation propagation.

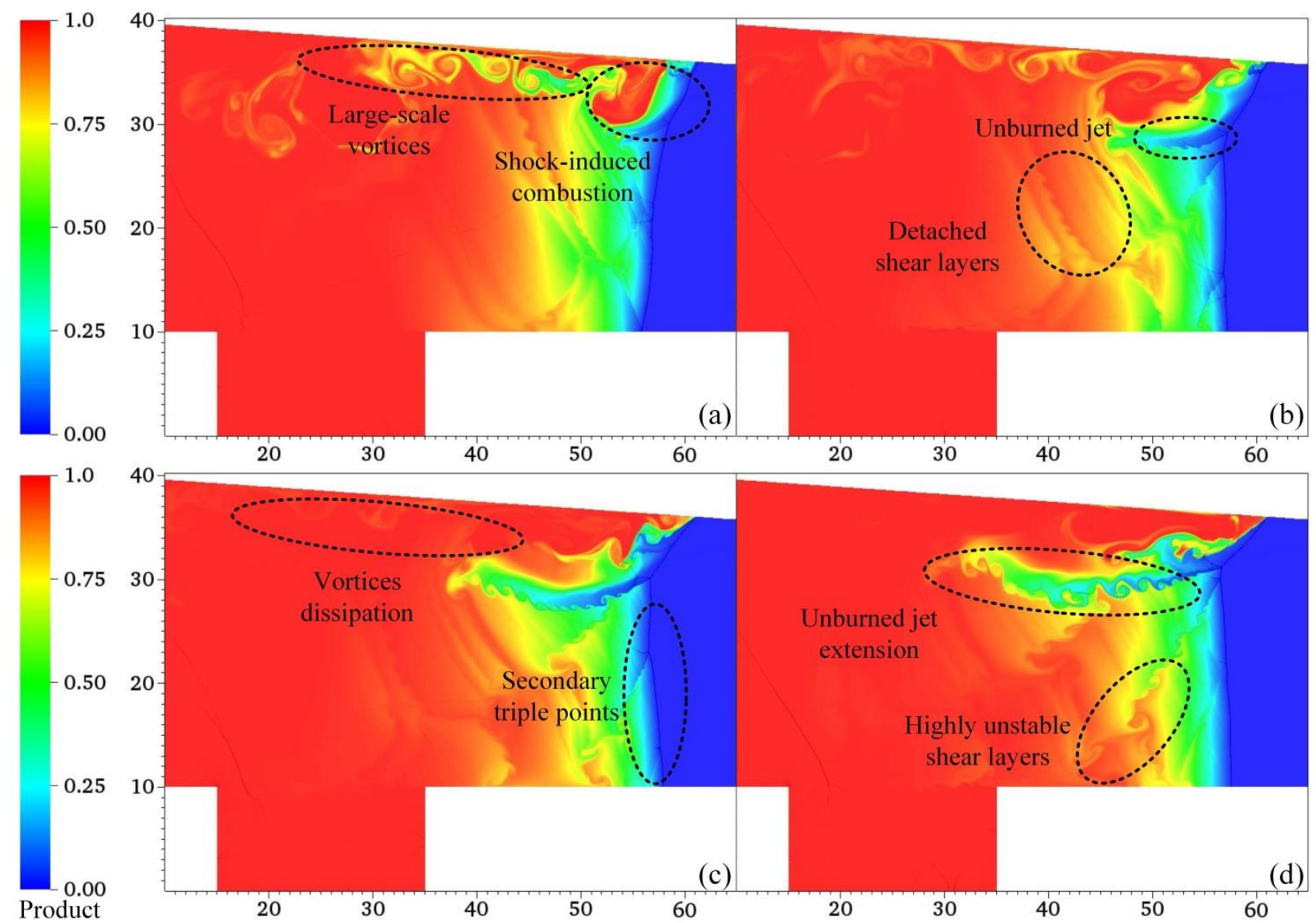

Fig. 5 Quasi-steady detonation propagation using product mass fraction contours, (a) $t=730 \mu \mathrm{s}$, (b)

$$
t=740 \mu \mathrm{s}, \text { (c) } t=750 \mu \mathrm{s} \text {, (d) } t=760 \mu \mathrm{s} .
$$

\subsection{Stabilization mechanism}


In supersonic flows through straight channels, the detonation wave begins its attenuation process associated with continuous backward propagation when the influence of the hot jet is dissipated (Cai et al. 2018b). However, as illustrated above in Fig. 5, the detonation wave realizes quasi-steady detonation propagation in the supersonic model combustor. Therefore, it seems reasonable to assume that the supersonic model combustor can contribute to the realization of detonation stabilization. In order to differentiate the effect of the expanding wall from that of the cavity in the supersonic model combustor on the quasi-steady propagation of the detonation wave, two comparison cases are studied: Model 1 considers a straight channel with a cavity and Model 2 employs an expanding channel without a cavity while the other conditions are remained the same.

Fig. 6 shows the detonation structures for both models after the shutdown of the hot jet at $t=310 \mu \mathrm{s}$. For Model 1, two triple points are clearly observed both in Figs.6(a) and 6(b). After the triple point reflections, detached shear layers are generated in Fig. 6(b). Compared with Fig. 5, no unburned jet is produced. Therefore, the flow field seems to be more laminar due to the absence of highly unstable shear layers. For Model 2 in Fig. 6(c), an unburned jet associated with the shear layer is generated because of the Prandtl-Meyer expansion.

As a result of the Mach reflection configuration with the respect to the plane of reflection, the slip line emanating from the primary triple point is redirected forward, thus forming a jet tangential to the expanding wall (Shi et al. 2019). This jet shoots forward along the wall towards the detonation front and gradually rolls up to form a vortex, resulting in the formation of the detonation bifurcation. With further evolution, a slight tripe point reflection is formed, and the shear layer along the unburned jet is further extended and becomes highly unstable, as shown in Fig. 6(d). Compared with that in Fig. 5, the main difference is that fewer triple points are generated together with relatively weaker strength of the detonation front using the pressure ratio (approximately $5 \%$ lower). 

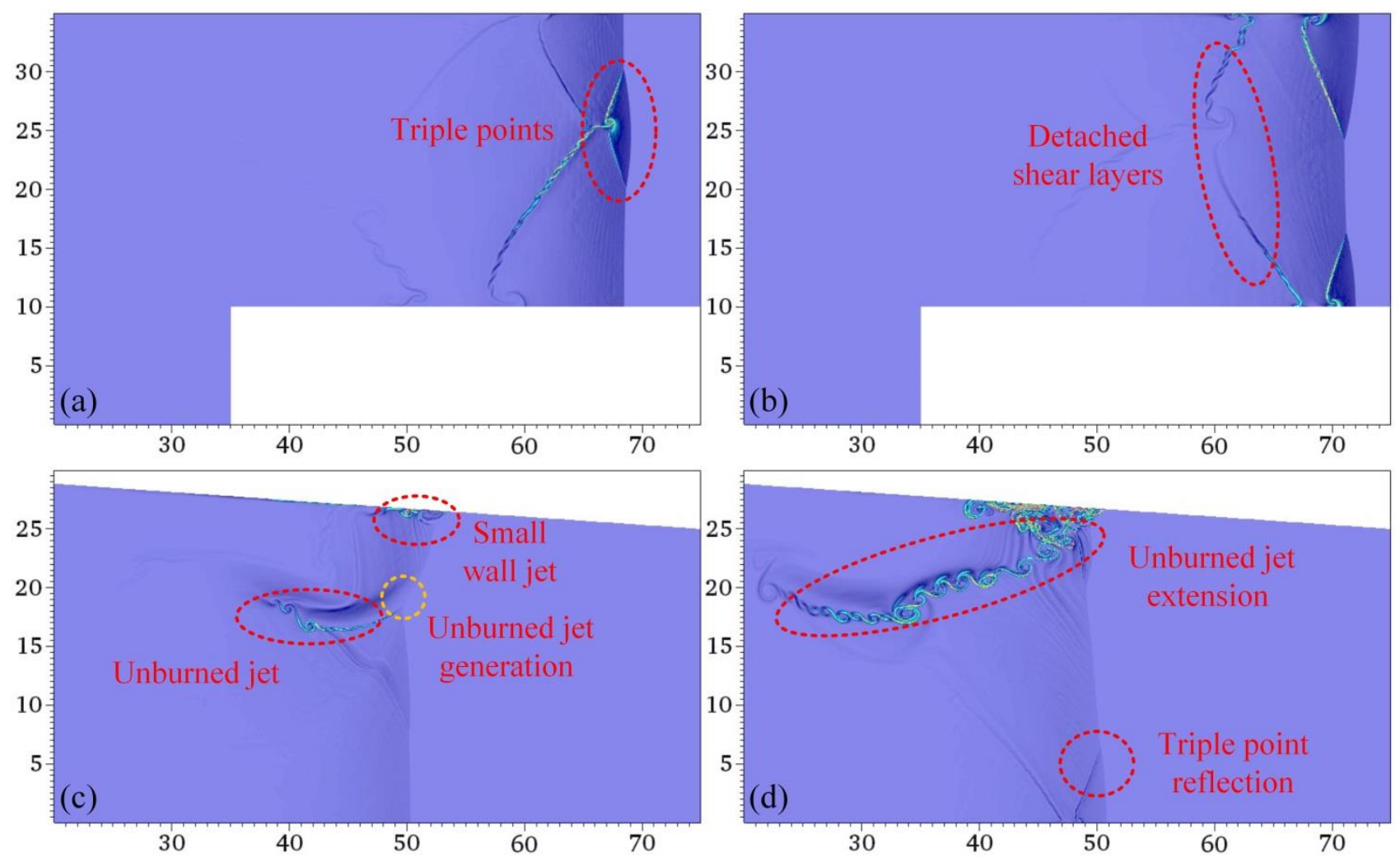

Fig. 6 Numerical species Schlieren showing detonation structures; Model 1: (a) $t=370 \mu$ s , (b)

$$
t=390 \mu \mathrm{s}, \text { Model 2: (c) } t=370 \mu \mathrm{s}, \text { (d) } t=390 \mu \mathrm{s} \text {. }
$$

Fig. 7 shows the comparison among the supersonic model combustor, Model 1 and Model 2. In Fig. 7(a), the trajectories of the detonation fronts are illustrated. It is seen that for the model combustor, after reaching the farthest position at about $t=400 \mu \mathrm{s}$. The detonation undergoes a slight backward propagation together with limited attenuation. Then, the trajectory curve globally begins to exhibit a plateau associated with periodical oscillations with an approximate period of $\Delta T=30 \mu \mathrm{s}$. This further verifies that a fluctuating quasi-steady propagation of detonation is eventually realized in the model combustor. For Model 1, after the shutdown of the hot jet, the trajectory exhibits periodical oscillations, but presents an overall constant slope, suggesting the eventual overdriven state of detonation. Note that, if the straight channel is long enough, eventually, the detonation wave should also relax back to the CJ state and stabilize at a farther location. The relative velocity of the forward propagation, represented by the constant slope, is calculated as 
$\Delta v=161.7 \mathrm{~m} / \mathrm{s}$ together with the corresponding overdrive degree of $f=1.21\left(f=\left(\frac{V_{C J}+\Delta v}{V_{C J}}\right)^{2}\right)$. The oscillation period is evaluated as $\Delta T_{\text {Model } 1}=30.4 \mu \mathrm{s}$, which is nearly the same as in the supersonic model combustor. This also indicates that the expanding wall does not have a significant influence on detonation oscillations. The trajectory in Model 2 illustrates a different trend compared to that in Model 1. After the shutdown of the hot jet, although the slope of the trajectory curve initially maintains a positive value, it turns negative at $t=340 \mu$ s after going through a short period of attenuation. This implies that the forward propagation of the detonation changes quickly to a backward propagation under this condition.
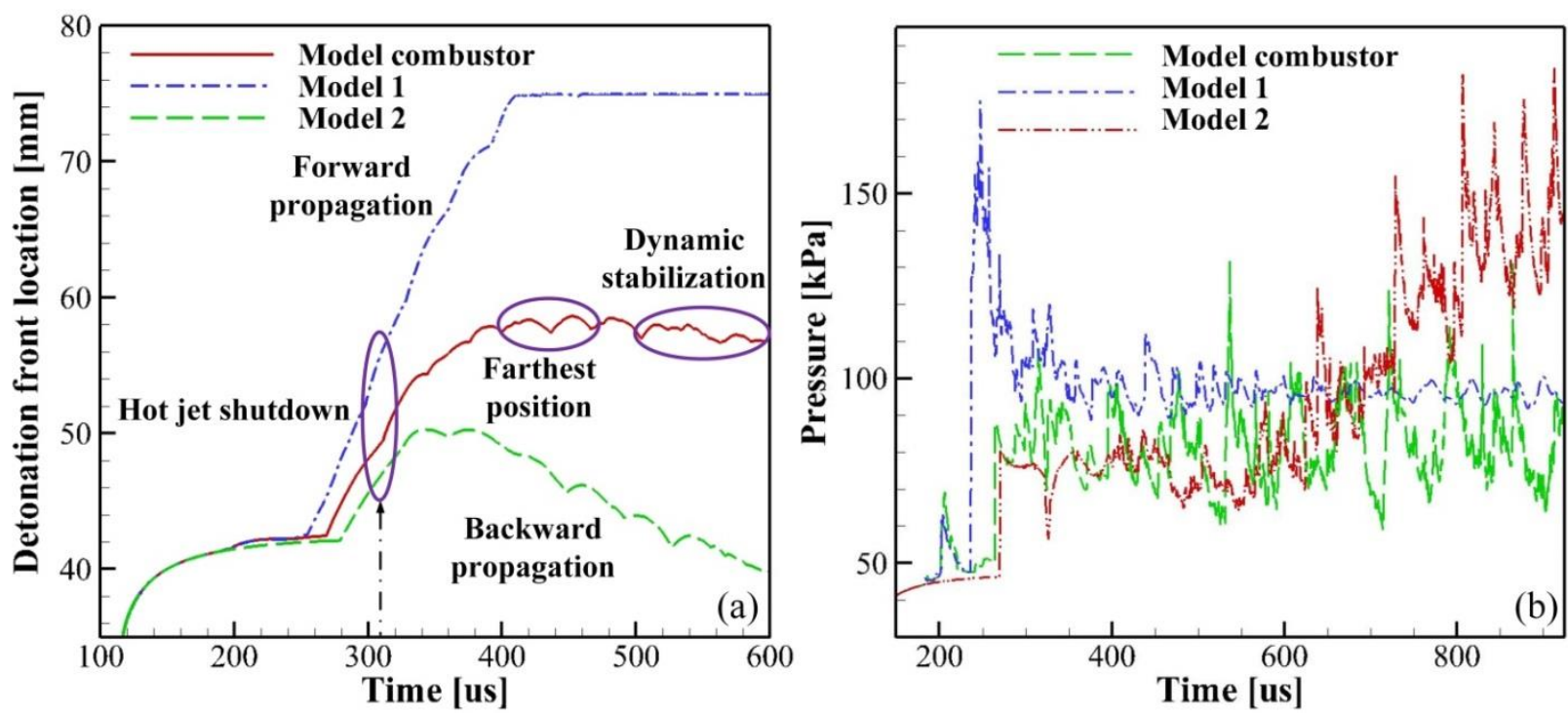

Fig. 7 (a) The trajectories of the detonation fronts and (b) Pressure oscillations at the same position in the main flow; Model 1: straight channel with a cavity and Model 2: expanding channel without a cavity.

It has been demonstrated (Cai et at. 2018c) that in supersonic flows the acoustic wave produced by the subsonic combustion in the cavity can accelerate detonation propagation after crossing through a subsonic channel in the vicinity of the cavity edge, which can result in the generation of an overdriven detonation. This is also true in the supersonic model combustor, as demonstrated in Fig. 
7(a). In the expanding channel, due to the lateral mass divergence resulting from the expanding wall, and the periodical formation and rapid consumption of the unburned jet resulting from the PrandtlMeyer expansion fan, the detonation wave can be maintained almost in the same position.

Fig. 7(b) illustrates pressure oscillations in the main flow, which are calculated at the same position. For the model combustor and Model 1, pressures are highly oscillating during the initial period up to $300 \mu \mathrm{s}$. Nevertheless, the amplitude of pressure oscillation in the model combustor is significantly lower than that in Model 1. The peak pressure in the model combustor is approximately $60 \%$ of that in Model 1, mainly due to the Prandtl-Meyer expansion fan. Because of the detonation stabilization in the model combustor, the pressure oscillation can maintain almost the same amplitude. However, for Model 1, due to the forward propagation of the overdriven detonation, the pressure is decreased quickly at the beginning and gradually reaches a relative periodic oscillation. For the model combustor and Model 2, the pressure gradually begins to oscillate significantly during the backward detonation propagation, although no obvious pressure oscillation is observed in Model 2 owing to the absence of the cavity.

It is indicated that the cavity in the supersonic flow could enhance pressure oscillations, which further facilitate the forward propagation of the detonation. On the other hand, the Prandtl-Meyer expansion fan resulting from the expanding wall can suppress pressure oscillations leading to detonation attenuation and failure. Therefore, in the supersonic model combustor consisting of the cavity and the expanding wall, the quasi-steady propagation of detonation can be properly realized as a result of two opposing effects.

\subsection{Effects of the incoming velocity}

Understanding the characteristics of detonation stabilization under the condition of different 
incoming velocities during accelerating or decelerating is important for detonation physics and practical applications. Based on the case in Sec.3.1, two cases with different characteristic incoming velocities are considered here as shown in Fig. 8. Run 1 uses an inflow velocity lower than the CJ velocity (i.e. $0.95 V_{C J}$ ) and Run 2 adopts an inflow speed higher than the CJ velocity (i.e. $1.05 V_{C J}$ ).

For Run 1, Figs.8(a) and 8(b) show that the overall structure continues its forward propagation after the shutdown of the hot jet. As seen in Fig. 8(a) an unburned jet has just been generated and the triple point is propagating downward. After some time however, as seen in Fig. 8(b), the unburned jet extends significantly and the triple point turns to upward propagation upon collision with the lower wall. Only one triple point (no secondary triple points) is observed on the detonation front and no obvious vortices are generated along the unburned jet.

This results in the absence of slip lines emitted from the triple points, which is quite different from that of the quasi-steady propagation of detonation discussed in the previous section. Without interactions of slip lines with the unburned jet, the diffusion and mixing effects on the unburned jet are suppressed, hence slowing down the consumption rate of the unburned jet and eventually leading to its obvious extension (Mahmoudi et al. 2014; Mazaheri, Mahmoudi \& Radulescu 2012). This indicates that when the incoming velocity is lower than the CJ value, supplementary methods should be utilized for realizing quasi-steady detonation propagation, which needs further investigation in the future work. 


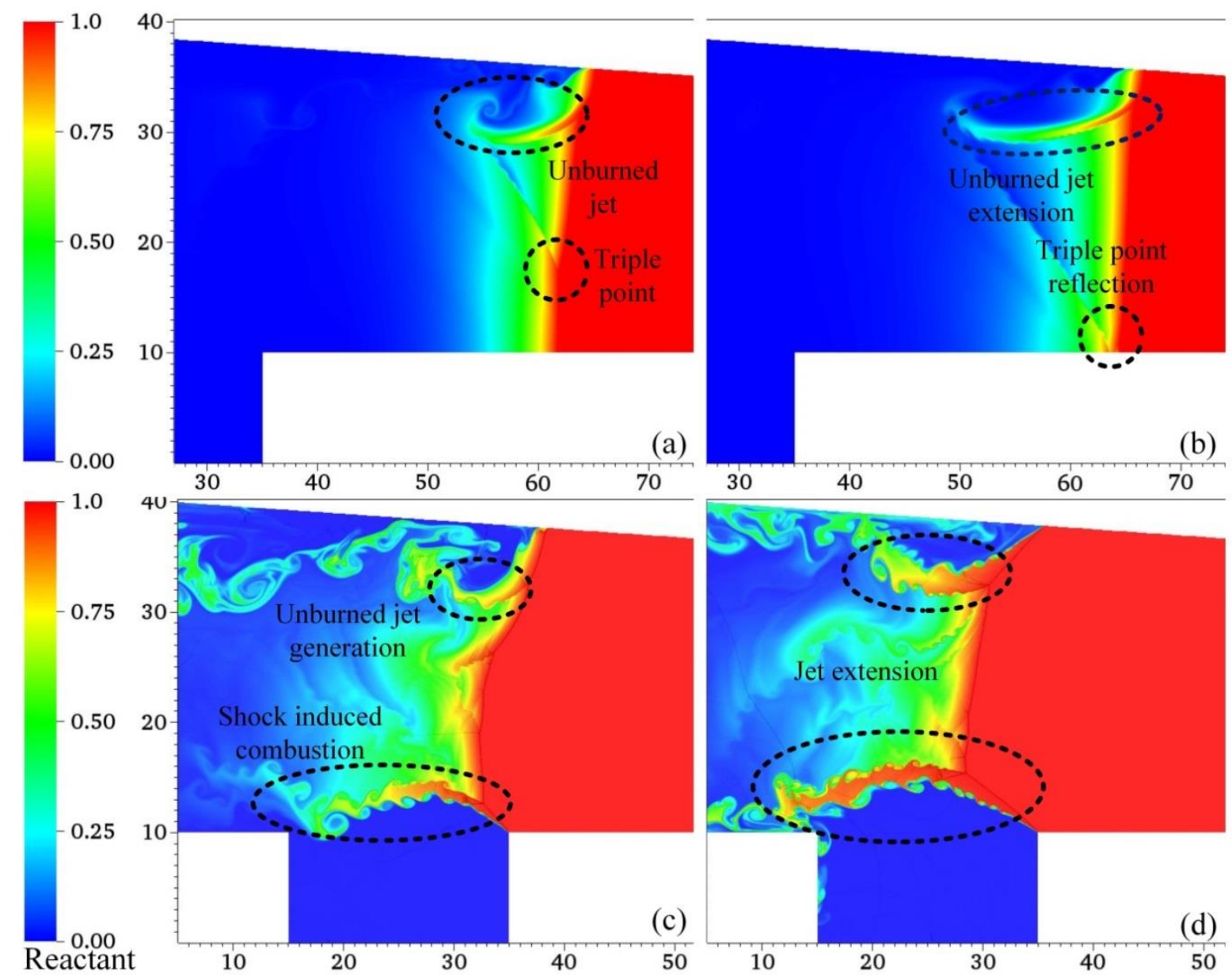

Fig. 8 Contours of reactant mass fraction illustrating detonation propagation; Run 1 (a) $t=325 \mu \mathrm{s}$ and (b) $t=335 \mu \mathrm{s}$, Run 2 (c) $t=615 \mu \mathrm{s}$ and (d) $t=755 \mu \mathrm{s}$.

For Run 2, the detonation wave quickly turns to backward propagation after the shutdown of the hot jet. Rather than stabilization during the backward propagation, the detonation wave continues the backward propagation until reaching the cavity. The configuration consists of a detonation wave in the central flow accompanied by two unburned jets. Fig. 8(c) illustrates the detonation wave interactions both with the cavity and the expanding wall. When the detonation wave passes over the cavity, an oblique shock wave associated with an unburned jet is generated within the detonation front. The oblique shock wave is separated from the reaction front, indicating the formation of oblique shock-induced combustion. This is a typical structure of detonation bifurcation resulting from the detonation wave interaction with the cavity. In addition to pressure oscillations, the cavity 
permits to anchor a shear layer at the leading edge which introduces a large reacting surface for the unburned jet. It has been demonstrated that the diffusion and mixing effect resulting from the physical viscosity can facilitate the consumption of unburned pockets behind the detonation front, thus accelerating the heat release rate (Radulescu et al. 2007). Due to the diffusion and mixing effects through the large reacting surface with neighboring burned gases, the generated unburned jet can be consumed effectively via which the chemical heat can be released rapidly. At this position, detonation bifurcation is developed underneath the expanding wall due to the generation of the wall jet, resulting in the generation of another unburned jet, as shown in Fig. 8(d). This structure mainly consists of one detonation front in the mainstream and two oblique shock-induced combustion waves associated with two unburned jets accompanying the detonation front.

Owing to adequate heat release resulting from the rapid consumption of the two unburned jets, which contributes significantly to the sustainment of detonation, the detonation begins its forward propagation once again, leading to the new generation of an overdriven detonation, as indicated in Fig. 9. However, the overdriven detonation begins to undergo an attenuation after it arrives at approximate $X=44 \mathrm{~mm}$. When the overdriven detonation propagates forward together with the gradual separation from the cavity, the unburned jet resulting from the detonation wave interaction with the cavity gradually decreases its size and eventually vanishes. This further reduces the heat release from the consumption of the unburned jet and finally results in the detonation attenuation. Subsequently, the detonation wave repeats the backward propagation and again the detonation bifurcation. This suggests that in the supersonic model combustor an oscillating periodic process is generated including four stages of forward propagation, detonation attenuation, backward propagation and detonation bifurcation. In the four stages, the detonation attenuation leads to backward propagation while the forward propagation results from detonation bifurcation, indicating 
the formation of detonation stabilization in the supersonic model combustor.

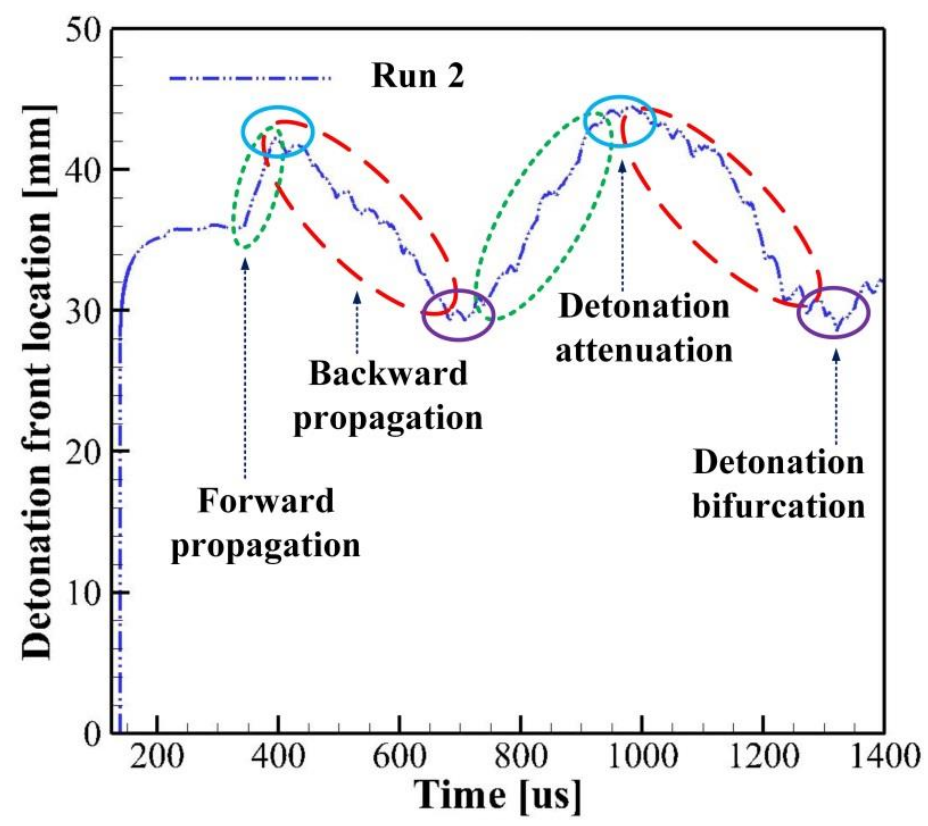

Fig. 9 The trajectory of the detonation front for Run 2.

\section{Summary and Conclusions}

In the present work, stabilization of a hydrogen/oxygen detonation in the supersonic model combustor is investigated by solving the reactive compressible Navier-Stokes equations and a onestep two-species reaction model using a hybrid sixth-order WENO-CD scheme within an SAMR framework.

A regularly fluctuating quasi-steady propagation of detonation can be realized in the supersonic model combustor when the detonation has propagated backwards after the shutdown of the hot jet. The overall configuration is in fact a detonation bifurcation consisting of a normal detonation wave and a shock-induced combustion front.

In this context, the realization of the quasi-steady propagation of detonation is due to the combined effects: (i) pressure oscillations generated in the cavity, which facilitate the detonation propagation; (ii) lateral mass divergence brought by the expanding wall which can lead to detonation 
attenuation, and an unburned jet associated with large-scale vortices resulting from a Prandtl-Meyer expansion fan which can contribute to the stabilization.

When the incoming velocity is lower than the CJ value, an overdriven detonation is generated; however, when the incoming velocity is larger than the CJ value, because of the formation of a periodic process of forward propagation, detonation attenuation, backward propagation and detonation bifurcation quasi-steady propagation of detonation can be realized effectively in the supersonic model combustor.

\section{Acknowledgements}

This work is supported by National Natural Science Foundation of China (No. 11702323), and National Postdoctoral Program for Innovative Talent (No. BX20180372).

\section{References}

BANE, S.P.M., ZIEGLER, J.L. \& SHEPHERD, J.E. 2010 Development of One-Step chemistry models for flame and ignition simulation, Report No. GALCITFM: 2010.002, California Institute of Technology.

BERGER, M. \& OLIER, J. 1984 Adaptive mesh refinement for hyperbolic partial differential equations. J. Comput. Phys. 53, 484-512.

CAI, X.D., DEITERDING, R., LIANG, J.H. \& MAHMOUDI, Y. 2017 Adaptive simulations of viscous detonations initiated by a hot jet using a high-order hybrid WENO-CD scheme. Proc. Combust. Inst. 36, 2725-2733.

CAI, X.D., LIANG, J.H., DEITERDING, R., MAHMOUDI, Y. \& SUN, M.B. 2018a Experimental and numerical investigations on propagating modes of detonations: Detonation 
wave/boundary layer interaction. Combust. Flame 190 201-215.

CAI, X.D., DEITERDING, R., LIANG, J.H., SUN, M.B. \& MAHMOUDI, Y. 2018b Diffusion and mixing effects in hot jet initiation and propagation of hydrogen detonations. J. Fluid Mech. 836 324-351.

CAI, X.D., DEITERDING, R., LIANG, J.H. Lin, Z.Y. \& Sun, M.B. 2018c Detonation interaction with cavity in supersonic combustible mixture. AIAA J. 56 2096-2102.

CAI, X.D., DEITERDING, R., LIANG, J.H., SUN, M.B. \& Dong, D.Z. 2019 Dynamic detonation stabilization in supersonic expanding channels. Phys. Rev. Fluids 4, 083201.

DEITERDING, R. 2003 Parallel adaptive simulation of multi-dimensional detonation structures, PhD thesis, Technical University of Cottbus, Cottbus, Germany.

DEITERDING, R. 2009 A parallel adaptive method for simulating shock-induced combustion with detailed chemical kinetics in complex domains. Comput. Struct. 87, 769-783.

DIMOTAKIS, P.E. 2005 Turbulent mixing. Annu. Rev. Fluid Mech. 37 329-356.

GAMEZO, V.N., OGAWA, T. \& ORAN, E.S. 2007 Numerical simulations of flame propagation and DDT in obstructed channels filled with hydrogen-air mixture. Proc. Combust. Inst. 31, 24632471.

GOTTLIEB, S., KETCHESON, D.I. \& SHU, C.W. 2009 High order strong stability preserving time discretizations. J. Sci. Comput. 38251.

GROGAN, K.P. \& IHME, M. 2015 Weak and strong ignition of hydrogen/oxygen mixtures in shocktube systems. Proc. Combust. Inst. 35, 2181-2189.

HILL, D.J. \& PULLIN, D.I. 2004 Hybrid tuned center-difference-WENO method for large eddy simulations in the presence of strong shocks. J. Comput. Phys. 194 435-450.

KAILASANATH, K. 2008 Review of propulsion applications of detonation waves. AIAA J. 38 
1698-1708.

KANG, S.H., LEE, Y.J., YANG, S.S., SMART, M.K. \& SURAWEERA, M.K. 2011 Cowl and Cavity Effects on Mixing and Combustion in Scramjet Engines. J. Propul. Power 27 11691177.

KAO, S. \& SHEPHERD, J.E. 2008 Numerical solution methods for control volume explosions and ZND detonation structure. GALCIT Rep. FM2006.007. California Institute of Technology, Pasadena, California.

KAPS, P. \& RENTROP, P. 1979 Generalized Runge-Kutta methods of order four with step size control for stiff ordinary differential equations. Numer. Math. 33, 55-68.

LOMBARDINI, M. 2008 Richtmyer-Meshkov instability in converging geometries, PhD thesis, California Institute of Technology, California, USA.

LU, F.K. \& BRAUN, E.M. 2014 Rotating detonation wave propulsion: Experimental challenges, modeling, and engine concepts. J. Propul. Power 30 1125-1142.

MAHMOUDI, Y., KARIMI, N., DEITERDING, R. \& EMAMI, S. 2014 Hydrodynamic instabilities in gaseous detonations: comparison of Euler, Navier-Stokes, and large-Eddy simulation. J. Propul. Power 30, 384-396.

MASSA, L. AUSTIN, J.M. \& JACKSON, T.L. 2007 Triple-point shear layers in gaseous detonation waves. J. Fluid Mech. 586 205-248.

MAXWELL, B.M., BHATTACHARJEE, R.R., LAU-CHAPDELAINE, S.S.M., FALLE, S.A.E.G., SHARPE, G.J. \& RADULESCU, M.I. 2017 Influence of turbulent fluctuations on detonation propagation. J. Fluid Mech. 818, 646-696.

MAZAHERI, K., MAHMOUDI, Y. \& RADULESCU, M.I. 2012 Diffusion and hydrodynamic instabilities in gaseous detonations. Combust. Flame 113, 2138-2154. 
ORAN, E.S. \& GAMEZO, V.N. 2007 Origins of the deflagration-to-detonation transition in gasphase combustion. Combust. Flame 148, 4-47.

PANTANO, C., DEITERDING, R., Hill, D.J. \& D.I. PULLIN. 2007 A low numerical dissipation patch-based adaptive mesh refinement method for large-eddy simulation of compressible flows. J. Comput. Phys. 221 63-87.

PENG, H., HUANG, Y., DEITERDING, R., LUAN, Z., XING, F. \& YOU, Y. 2018 Effects of jet in crossflow on flame acceleration and deflagration to detonation transition in methane-oxygen mixture. Combust. Flame 198 69-80.

POTTURI, A.S. \& EDWARDS, J.R. 2015 Large-eddy/Reynolds-averaged Navier-Stokes simulation of cavity-stabilized ethylene combustion. Combust. Flame 162 1176-1192.

RADULESCU, M.I. \& BORZOU, B. 2018 Dynamics of detonations with a constant mean flow divergence. J. Fluid Mech. 845 346-377.

RADULESCU, M.I. 2018 A detonation paradox: Why inviscid detonation simulations predict the incorrect trend for the role of instability in gaseous cellular detonations. Combust. Flame $\mathbf{1 9 5}$ $151-162$.

RADULESCU, M.I., SHARPE, G.J., LAW, C.K. \& LEE, J.H.S. 2007 The hydrodynamic structure of unstable cellular detonations. J. Fluid Mech. 580, 31-81.

RADULESCU, M.I., SHARPE, G.J., LEE, J.H.S., KIYANDA, C.B., HIGGINS, A.J. \& HANSON, R.K. 2005 The ignition mechanism in irregular structure gaseous detonations. Proc. Combust. Inst. 30 (2), 1859-1867.

ROMICK, C.M., ASLAM, T.D. \& POWERS, J.M. 2012 The effect of diffusion on the dynamics of unsteady detonations. J. Fluid Mech. 699, 453-464

SHEN, H. \& PARSANI, M. 2017 The role of multidimensional instabilities in direct initiation of 
gaseous detonations in free space. J. Fluid Mech 813, R4.

SHI, X.F., ZHU, Y.J., YANG, J.M. \& LUO, X.S. 2019 Mach stem deformation in pseudo-steady shock wave reflections, J. Fluid Mech. 861 407-421.

SUN, M.B., WANG, Z.G., LIANG, J.H. \& GENG, H. 2008 Flame characteristics in cupersonic combustor with hydrogen injection upstream of cavity flameholder. J. Propul. Power 24 688696.

TATMAN, B.J., ROCKWELL, R.D., GOYNE, C.P., MCDANILE, J.C. \& DONOHUE, J.M. 2013 Experimental study of vitiation effects on flameholding in a cavity flameholder. J. Propul. Power 29 417-423.

TENG, H.H., JIANG, Z.L. \& NG, H.D. 2014 Numerical study on unstable surfaces of oblique detonations. J. Fluid Mech 744, 111-128.

WANG, Y., HAN, W., DEITERDING, R. \& Chen, Z. 2018 Effects of disturbance on detonation initiation in $\mathrm{H}_{2}-\mathrm{O}_{2}-\mathrm{N}_{2}$ mixture. Phys. Rev. Fluids 3123201.

WOLAŃSKI, P. 2013 Detonative Propulsion. Proc. Combust. Inst. 34 125-158.

XIAO, H.H. \& ORAN, E.S. 2019 Shock focusing and detonation initiation at a flame front. Combust. Flame 203 397-406.

YEOM, H.W., SEO, B.G. \& SUNG, H.G. 2013 Numerical analysis of a scramjet engine with intake sidewalls and cavity flameholder. AIAA J. 51 1566-1575.

ZHANG, X.J., WEI, H.Q., ZHOU, L., CAI, X.D. \& DEITERDING, R. 2020 Relationship of flame propagation and combustion mode transition of end-gas based on pressure wave in confined space, Combust. Flame 214 371-386.

ZIEGLER, J.L., DEITERDING, R., SHEPHERD, J.E. \& PULLIN, D.I. 2011 An adaptive high-order hybrid scheme for compressive, viscous flows with detailed chemistry. J. Comput. Phys. 230, 
7598-7630. 